\title{
ON THE HAUTUS TEST FOR EXPONENTIALLY STABLE $C_{0}$-GROUPS*
}

\author{
BIRGIT JACOB ${ }^{\dagger}$ AND HANS ZWART
}

\begin{abstract}
For finite-dimensional systems the Hautus test is a well-known and easy checkable condition for observability. Russell and Weiss [SIAM J. Control Optim., 32 (1994), pp. 1-23] suggested an infinite-dimensional version of the Hautus test, which is necessary for exact observability and sufficient for approximate observability of exponentially stable systems. In this paper it is shown that this Hautus test is sufficient for exact observability of certain exponentially stable systems generated by a $C_{0}$-group, and it is proved that the Hautus test is in general not sufficient for approximate observability of strongly stable systems even if the system is modeled by a contraction semigroup and the observation operator is bounded.
\end{abstract}

Key words. infinite-dimensional systems, unbounded observation operator, exact observability, Hautus test

AMS subject classifications. 47D06, 93C25, 93B07, 93B28

DOI. $10.1137 / 080724733$

1. Introduction and main results. We consider the abstract system

$$
\begin{aligned}
& \dot{x}(t)=A x(t), \quad x(0)=x_{0}, \quad t \geq 0, \\
& y(t)=C x(t), \quad t \geq 0,
\end{aligned}
$$

on a Hilbert space $H$. Here $A$ is the infinitesimal generator of a $C_{0}$-semigroup $(T(t))_{t \geq 0}$ and by the solution of (1) we mean $x(t)=T(t) x_{0}$, the weak solution. If $C$ is a bounded linear operator from $H$ to a second Hilbert space $Y$, then it is straightforward to see that $y(\cdot)$ in $(2)$ is well defined and continuous. However, in many PDEs rewritten in the form (1)-(2), $C$ is only a bounded operator from $D(A)$, the domain of $A$, to $Y$, although the output $y$ is a well-defined (locally) square integrable function. In the following, $C$ will always be a bounded operator from $D(A)$, equipped with the graph norm, to $Y$. If the output is square integrable on the time interval $(0, \infty)$, then $C$ is called an infinite-time admissible observation operator for $(T(t))_{t \geq 0}$; see Weiss [15] and Jacob and Partington [5]. Using the uniform boundedness theorem, we see that the observation operator $C$ is infinite-time admissible if and only if there exists a constant $L>0$ such that

$$
\int_{0}^{\infty}\left\|C T(t) x_{0}\right\|^{2} d t \leq L\left\|x_{0}\right\|^{2}, \quad x_{0} \in D(A) .
$$

Note that the first norm is in $Y$, whereas the second norm is in $H$. In the following, we will always assume that $C$ is an infinite-time admissible observation operator for $(T(t))_{t \geq 0}$. We introduce the following observability concepts.

\footnotetext{
* Received by the editors May 20, 2008; accepted for publication (in revised form) January 20, 2009; published electronically April 1, 2009.

http://www.siam.org/journals/sicon/48-3/72473.html

${ }^{\dagger}$ Institut für Mathematik, Universität Paderborn, Warburger Straße 100, D-33098 Paderborn, Germany (jacob@math.uni-paderborn.de).

¥Department of Applied Mathematics, Faculty of Electrical Engineering, Mathematics and Computer Science, University of Twente, P.O. Box 217, 7500 AE Enschede, The Netherlands (h.j.zwart@ math.utwente.nl).
} 
Definition 1.1. The pair $(A, C)$ is called exactly observable in time $t_{0}>0$ if there exists a constant $\kappa_{t_{0}}>0$ such that

$$
\kappa_{t_{0}} \int_{0}^{t_{0}}\left\|C T(t) x_{0}\right\|^{2} d t \geq\left\|x_{0}\right\|^{2}, \quad x_{0} \in D(A) .
$$
that

The pair $(A, C)$ is called exactly observable if there exists a constant $\kappa>0$ such

$$
\kappa \int_{0}^{\infty}\left\|C T(t) x_{0}\right\|^{2} d t \geq\left\|x_{0}\right\|^{2}, \quad x_{0} \in D(A) .
$$

The pair $(A, C)$ is called final state observable if there exist constants $\kappa, t_{0}>0$ such that

$$
\kappa \int_{0}^{\infty}\left\|C T(t) x_{0}\right\|^{2} d t \geq\left\|T\left(t_{0}\right) x_{0}\right\|^{2}, \quad x_{0} \in D(A) .
$$

The pair $(A, C)$ is called approximately observable if

$$
\int_{0}^{\infty}\left\|C T(t) x_{0}\right\|^{2} d t>0, \quad x_{0} \in D(A) \backslash\{0\} .
$$

Clearly, approximate observability and final state observability are weaker concepts than exact observability, whereas exact controllability in time $t_{0}$ is a stronger concept. For $C_{0}$-groups, the concepts of exact observability and final state observability are equivalent notions. In Russell and Weiss [14] it is shown that a necessary condition for exact observability of exponentially stable systems is the following version of the Hautus test:

There exists a constant $m>0$ such that for every $s \in \mathbb{C}_{-}$and every $x \in D(A)$,

$$
\|(s I-A) x\|^{2}+|\operatorname{Re} s|\|C x\|^{2} \geq m|\operatorname{Re} s|^{2}\|x\|^{2} .
$$

Here $\mathbb{C}_{-}$denotes the open left half plane. The Hautus test (HT) is sufficient for approximate observability of exponentially stable systems [14] and for polynomially stable systems [6]. Further, (HT) is sufficient for exact observability of strongly stable Riesz-spectral systems with finite-dimensional output spaces [7], for exponentially stable systems with $A$ bounded on $H$ [14], and for exponentially stable systems if the constant $m$ in (HT) equals 1 [3]; a short proof of this last result can be found in section 4. However, in general (HT) is not sufficient for exponentially stable systems [9]. We refer the reader to Russell and Weiss [14] and Jacob and Zwart [7, 8] for more information on (HT). Related to (HT) is an equivalent condition for exact observability of groups of unitary operators; see our section 2, [16], and [10].

In this paper, we show in particular that (HT) is sufficient for exponentially stable systems with a normal $C_{0}$-group, and we prove that $(\mathrm{HT})$ is in general not sufficient for strongly stable systems even if the operator $C$ is bounded and $A$ generates a contraction semigroup. More precisely, the main results of this paper are as follows.

TheOREm 1.2. Let $A$ be the generator of a $C_{0}$-group $(T(t))_{t \in \mathbb{R}}$ satisfying

$$
M_{1} e^{\alpha_{1} t}\left\|x_{0}\right\| \leq\left\|T(t) x_{0}\right\| \leq M_{2} e^{\alpha_{2} t}\left\|x_{0}\right\|
$$


for every $t \geq 0$ and $x_{0} \in H$ and some constants $M_{1}, M_{2}>0$ and $\alpha_{1}<\alpha_{2}<0$. Further, we assume that (HT) is satisfied for $s=\alpha_{2}+i \omega, \omega \in \mathbb{R}$.

If

$$
\frac{\alpha_{2}-\alpha_{1}}{\left|\alpha_{2}\right|}<\frac{\sqrt{m} M_{1}}{4 e M_{2}}
$$

then the pair $(A, C)$ is exactly observable in time $t_{0}=\left(\alpha_{2}-\alpha_{1}\right)^{-1}$.

THEOREM 1.3. Let $A$ be the generator of an exponentially stable normal $C_{0}$ semigroup $(T(t))_{t \geq 0}$. Then (HT) is sufficient for final state observability.

Corollary 1.4. Let $A$ be the generator of an exponentially stable normal $C_{0^{-}}$ group $(T(t))_{t \in \mathbb{R}}$. Then $(H T)$ is equivalent to exact observability.

We recall that the $C_{0}$-semigroup $(T(t))_{t \geq 0}$ is said to be strongly stable if $\left\|T(t) x_{0}\right\| \rightarrow$ 0 as $t \rightarrow 0$ for every $x_{0} \in H$.

THEOREM 1.5. There exists a strongly stable contraction semigroup on a Hilbert space with generator $A$ such that

$$
\|(s I-A) x\| \geq m|\operatorname{Re} s|\|x\|, \quad \operatorname{Re} s<0, x \in D(A) .
$$

In particular, the pair $(A, 0)$ satisfies $(H T)$. Clearly the zero operator is infinite-time admissible for the semigroup, but the pair $(A, 0)$ is not approximately observable.

We proceed as follows. In section 2 the Fourier transform is used as in Miller [10] to prove a more general version of both Miller's result on unitary groups and Theorem 1.2. Section 3 is devoted to normal $C_{0}$-semigroups. In particular, it is shown that (HT) is sufficient for exponentially stable systems generated with a normal $C_{0}$-group. Finally, in section 4 , we prove that (HT) with $m=1$ implies exact observability for strongly stable systems. Furthermore, the proof of Theorem 1.5 is presented.

2. The Hautus test for $\boldsymbol{C}_{\mathbf{0}}$-groups. In order to prove (HT) for a class of $C_{0}$-groups, we need the following lemma, which is taken from Opic and Kufner [12, page 94].

Lemma 2.1. Let $t_{0}>0$ and $\rho_{1}, \rho_{2} \in \mathbb{R}$. Then there exists a constant $\gamma>0$ such that

$$
\gamma \int_{0}^{t_{0}}|\chi(t)|^{2} e^{2 \rho_{1} t} d t \leq \int_{0}^{t_{0}}|\dot{\chi}(t)|^{2} e^{2 \rho_{2} t} d t
$$

for every $\chi \in C_{c}^{\infty}\left(0, t_{0}\right)$. Here $C_{c}^{\infty}\left(0, t_{0}\right)$ denotes the set of all the functions in $C^{\infty}\left(0, t_{0}\right)$ with compact support. The best possible constant $\gamma=\gamma\left(t_{0}, \rho_{1}, \rho_{2}\right)$ satisfies

$$
\frac{1}{12} \delta^{-1} \leq \gamma\left(t_{0}, \rho_{1}, \rho_{2}\right) \leq 2 \delta^{-1}
$$

with

$$
\delta:=\sup \left\{\int_{a}^{b} e^{2 \rho_{1} t} d t \cdot \min \left[\int_{0}^{a} e^{-2 \rho_{2} t} d t, \int_{b}^{t_{0}} e^{-2 \rho_{2} t} d t\right]\right\},
$$

where the supremum is taken over all pairs $(a, b)$ with $0<a<b<t_{0}$.

An easy calculation shows that $\gamma\left(t_{0}, \rho_{1}, \rho_{2}\right)=\gamma\left(1, \rho_{1} t_{0}, \rho_{2} t_{0}\right) / t_{0}^{2}$. Proposition 2.2 is the most general version of Theorem 1.2.

Proposition 2.2. Assume that there are constants $\alpha_{1}, \alpha_{2}, \beta \in \mathbb{R}$ with $\alpha_{1} \leq \alpha_{2}$ and $M_{1}, M_{2}, m_{1}, m_{2}, t_{0}>0$, and an element $x_{0} \in D(A)$ satisfying 
(i) $M_{1} e^{\alpha_{1} t}\left\|x_{0}\right\| \leq\left\|T(t) x_{0}\right\| \leq M_{2} e^{\alpha_{2} t}\left\|x_{0}\right\|$ for $0 \leq t \leq t_{0}$;

(ii) $\|x\|^{2} \leq m_{1}\|((\beta+i \omega) I-\bar{A}) x\|^{2}+m_{2}\|C x\|^{2}$ for every $x \in D(A)$ and all $\omega \in \mathbb{R}$;

(iii) $\gamma:=\gamma\left(t_{0}, \alpha_{1}-\beta, \alpha_{2}-\beta\right)<\frac{M_{1}^{2}}{m_{1} M_{2}^{2}}$, where $\gamma$ is as in (6).

Then for every $\varepsilon \in\left(0, t_{0}^{2} M_{1}^{2} /\left(m_{1} M_{2}^{2}\right)-t_{0}^{2} \gamma\right)$ there exists a constant $C_{\varepsilon}>0$ such that

$$
\frac{C_{\varepsilon} m_{2}}{t_{0}\left(M_{1}^{2}-\left(\gamma+\varepsilon / t_{0}^{2}\right) m_{1} M_{2}^{2}\right)} \int_{0}^{t_{0}}\left\|C T(t) x_{0}\right\|^{2} d t \geq\left\|x_{0}\right\|^{2} .
$$

The constant $C_{\varepsilon}$ depends only on $\varepsilon,\left(\alpha_{1}-\beta\right) t_{0},\left(\alpha_{2}-\beta\right) t_{0}$, and $\beta t_{0}$.

Proof. Let $\varepsilon \in\left(0, t_{0}^{2} M_{1}^{2} /\left(m_{1} M_{2}^{2}\right)-t_{0}^{2} \gamma\right)$. We choose $\chi_{1} \in C_{c}^{\infty}(0,1)$ such that

$$
\begin{aligned}
& {\left[\gamma\left(1,\left(\alpha_{1}-\beta\right) t_{0},\left(\alpha_{2}-\beta\right) t_{0}\right)+\varepsilon\right] \int_{0}^{1}\left|\chi_{1}(t)\right|^{2} e^{2\left(\alpha_{1}-\beta\right) t_{0} t} d t} \\
& \geq \int_{0}^{1}\left|\dot{\chi}_{1}(t)\right|^{2} e^{2\left(\alpha_{2}-\beta\right) t_{0} t} d t .
\end{aligned}
$$

Note that $\chi_{1}$ depends only on $\varepsilon,\left(\alpha_{1}-\beta\right) t_{0}$, and $\left(\alpha_{2}-\beta\right) t_{0}$. The existence of $\chi_{1}$ is guaranteed by Lemma 2.1. Defining

$$
\chi(t):= \begin{cases}\chi_{1}\left(t / t_{0}\right), & t \in\left[0, t_{0}\right], \\ 0, & t \in \mathbb{R} \backslash\left[0, t_{0}\right],\end{cases}
$$

we obtain

$$
\left(\gamma+\varepsilon / t_{0}^{2}\right) \int_{0}^{t_{0}}|\chi(t)|^{2} e^{2\left(\alpha_{1}-\beta\right) t} d t \geq \int_{0}^{t_{0}}|\dot{\chi}(t)|^{2} e^{2\left(\alpha_{2}-\beta\right) t} d t
$$

We define

$$
z(t):= \begin{cases}\chi(t) e^{-\beta t} T(t) x_{0}, & t \geq 0, \\ 0, & t<0 .\end{cases}
$$

By the choice of $\chi$ and $x_{0}$, the function $z$ is differentiable on $\mathbb{R}$ with

$$
\dot{z}(t)=\dot{\chi}(t) e^{-\beta t} T(t) x_{0}+\chi(t) e^{-\beta t} T(t)[-\beta I+A] x_{0}, \quad t \geq 0,
$$

and $\dot{z}(t)=0$ if $t<0$. Defining $f(t):=\dot{\chi}(t) e^{-\beta t} T(t) x_{0}$ for $t \geq 0$, and $f(t)=0$ otherwise, and using the Fourier transform, we get

$$
i \omega \hat{z}(i \omega)=\hat{f}(i \omega)+(-\beta I+A) \hat{z}(i \omega), \quad \omega \in \mathbb{R},
$$

or equivalently,

$$
((\beta+i \omega) I-A) \hat{z}(i \omega)=\hat{f}(i \omega), \quad \omega \in \mathbb{R} .
$$

Replacing $x$ in (ii) by $\hat{z}(i \omega)$ and using (10), we have

$$
m_{1}\|\hat{f}(i \omega)\|^{2}+m_{2}\|C \hat{z}(i \omega)\|^{2} \geq\|\hat{z}(i \omega)\|^{2} .
$$

We integrate this inequality over $\omega$ from $-\infty$ to $\infty$ and we use Parseval's equality to obtain

$$
m_{1} \int_{-\infty}^{\infty}\|f(t)\|^{2} d t+m_{2} \int_{-\infty}^{\infty}\|C z(t)\|^{2} d t \geq \int_{-\infty}^{\infty}\|z(t)\|^{2} d t
$$


This implies

$$
\begin{aligned}
m_{2} \sup _{0 \leq t \leq t_{0}} e^{-2 \beta t}|\chi(t)|^{2} \int_{0}^{t_{0}}\left\|C T(t) x_{0}\right\|^{2} d t \\
\quad \geq m_{2} \int_{0}^{t_{0}}\|C z(t)\|^{2} d t \\
\quad \geq \int_{0}^{t_{0}}|\chi(t)|^{2}\left\|T(t) x_{0}\right\|^{2} e^{-2 \beta t} d t-m_{1} \int_{0}^{t_{0}}|\dot{\chi}(t)|^{2}\left\|T(t) x_{0}\right\|^{2} e^{-2 \beta t} d t \\
\quad \geq\left[M_{1}^{2} \int_{0}^{t_{0}}|\chi(t)|^{2} e^{2\left(\alpha_{1}-\beta\right) t} d t-m_{1} M_{2}^{2} \int_{0}^{t_{0}}|\dot{\chi}(t)|^{2} e^{2\left(\alpha_{2}-\beta\right) t} d t\right]\left\|x_{0}\right\|^{2} \\
\\
\quad \geq\left[\left(M_{1}^{2}-\left(\gamma+\varepsilon / t_{0}^{2}\right) m_{1} M_{2}^{2}\right) \int_{0}^{t_{0}}|\chi(t)|^{2} e^{2\left(\alpha_{1}-\beta\right) t} d t\right]\left\|x_{0}\right\|^{2} .
\end{aligned}
$$

By our assumption on $\gamma$ and $\varepsilon$, we have that the constant in front of the last integral is positive. Thus the above inequality is equivalent to

$$
\frac{m_{2}}{t_{0}\left(M_{1}^{2}-\left(\gamma+\varepsilon / t_{0}^{2}\right) m_{1} M_{2}^{2}\right)} \frac{\sup _{0 \leq t \leq 1}\left[e^{-2 \beta t_{0} t}\left|\chi_{1}(t)\right|^{2}\right]}{\int_{0}^{1}\left|\chi_{1}(t)\right|^{2} e^{2\left(\alpha_{1}-\beta\right) t_{0} t} d t} \int_{0}^{t_{0}}\left\|C T(t) x_{0}\right\|^{2} d t \geq\left\|x_{0}\right\|^{2} .
$$

Now the statement of the proposition follows with

$$
C_{\varepsilon}=\frac{\sup _{0 \leq t \leq 1}\left[e^{-2 \beta t_{0} t}\left|\chi_{1}(t)\right|^{2}\right]}{\int_{0}^{1}\left|\chi_{1}(t)\right|^{2} e^{2\left(\alpha_{1}-\beta\right) t_{0} t} d t} .
$$

The following remark is needed for the proof of Theorems 1.2 and 1.3.

Remark 2.3. If we choose $\alpha_{1}<\beta=\alpha_{2}, m_{1}=\left(m \beta^{2}\right)^{-1}, m_{2}=(m|\beta|)^{-1}$, and $t_{0}=\left(\alpha_{2}-\alpha_{1}\right)^{-1}$, then from the lines following Lemma 2.1 we have that $\gamma=$ $\left(\alpha_{2}-\alpha_{1}\right)^{2} \gamma(1,-1,0)$. So it remains to estimate $\gamma(1,-1,0)$. Choosing in (7) $a=1 / 4$ and $b=3 / 4$, we have that

$$
\delta \geq \frac{1}{4} \int_{\frac{1}{4}}^{\frac{3}{4}} e^{-2 t} d t \geq \frac{1}{8} e^{-\frac{3}{2}}
$$

Combining this with (6), we find that $\gamma(1,-1,0) \leq 16 e \sqrt{e}<16 e^{2}$. Hence $\gamma \leq$ $16 e^{2}\left(\alpha_{2}-\alpha_{1}\right)^{2}$.

Now using the value of $m_{1}$ and $m_{2}$, we see that the third assumption of Proposition 2.2 is satisfied if

$$
16 e^{2} \frac{\left(\alpha_{2}-\alpha_{1}\right)^{2}}{\alpha_{2}^{2}}<\frac{m M_{1}^{2}}{M_{2}^{2}}
$$

and (8) implies

$$
\frac{\alpha_{2}-\alpha_{1}}{\left|\alpha_{2}\right|} \frac{C_{\varepsilon}}{m M_{1}^{2}-\frac{\left(\alpha_{2}-\alpha_{1}\right)^{2}}{\alpha_{2}^{2}}\left(16 e^{2}+\varepsilon\right) M_{2}^{2}} \int_{0}^{t_{0}}\left\|C T(t) x_{0}\right\|^{2} d t \geq\left\|x_{0}\right\|^{2}
$$

for $\varepsilon \in\left(0, m M_{1}^{2} \alpha_{2}^{2} /\left(\left(\alpha_{2}-\alpha_{1}\right)^{2} M_{2}^{2}\right)-16 e^{2}\right)$. Note that $C_{\varepsilon}$ depends only on $\varepsilon$ and $\alpha_{2} /\left(\alpha_{2}-\alpha_{1}\right)$.

Proof of Theorem 1.2. This follows immediately from the previous remark.

Copyright (C) by SIAM. Unauthorized reproduction of this article is prohibited. 
As a corollary we obtain the following result.

Corollary 2.4 (see Miller [10]). Let $A$ be the generator of $C_{0}$-group $(T(t))_{t \geq 0}$ of unitary operators. Further, we assume that there exist constants $m, M>0$ such that

$$
\|x\|^{2} \leq m_{1}\|(A-i \omega) x\|^{2}+m_{2}\|C x\|^{2}
$$

holds for every $x \in D(A)$ and all $\omega \in \mathbb{R}$. Then the pair $(A, C)$ is exactly observable in time $t_{0}>\pi \sqrt{m_{1}}$.

Proof. We choose $\alpha_{1}=\alpha_{2}=\beta=0$ and $M_{1}=M_{2}=1$ in Proposition 2.2. Since

$$
\inf _{\phi \in C_{c}^{\infty}(0,1)} \frac{\int_{0}^{1} \dot{\phi}^{2}(t) d t}{\int_{0}^{1} \phi^{2}(t) d t}=\pi^{2},
$$

we have $\gamma(1,0,0)=\pi^{2}$ and thus $\gamma\left(t_{0}, 0,0\right)=\pi^{2} / t_{0}^{2}$. Applying Proposition 2.2, we obtain that the pair $(A, C)$ is exactly observable in time $t_{0}>\pi \sqrt{m_{1}}$.

3. The Hautus test for normal $C_{0}$-groups. In this section we assume that $A$ is a normal operator and that it generates an exponentially stable $C_{0}$-semigroup. Since $A$ is normal, there exists a measure $E$ on $\mathbb{C}$ such that

$$
A x_{0}=\int_{\mathbb{C}} \lambda d E(\lambda) x_{0} .
$$

Due to the fact that $A$ is the infinitesimal generator of an exponential stable $C_{0^{-}}$ semigroup, the spectral measure has no support on $\left\{\lambda \in \mathbb{C} \mid \operatorname{Re} \lambda>\alpha_{0}\right\}$ for some $\alpha_{0}<0$. The semigroup generated by $A$ has the expansion

$$
T(t) x_{0}=\int_{\mathbb{C}} e^{\lambda t} d E(\lambda) x_{0} .
$$

Furthermore, the norm of $T(t) x_{0}$ can be calculated by

$$
\left\|T(t) x_{0}\right\|^{2}=\int_{\mathbb{C}} e^{2 \operatorname{Re}(\lambda) t} d\left\|E(\lambda) x_{0}\right\|^{2} .
$$

Since Jensen's inequality plays an important role in our proof, we summarize it here. A proof can be found in Rudin [13].

LEMMA 3.1. Let $\mu$ be a positive measure on a set $\Omega$ with $\mu(\Omega)=1$. Let $f$ be a real function in $L^{1}(\mu)$ with $f(x)>0$ a.e. If $\phi$ is a convex function on $(0, \infty)$, then

$$
\phi\left(\int_{\Omega} f d \mu\right) \leq \int_{\Omega}(\phi \circ f) d \mu .
$$

The following lemma will be very useful for the proof of Theorem 1.3.

Lemma 3.2. For $x_{0} \in D(A)$ with norm one, we define the function $g:[0, \infty) \rightarrow \mathbb{R}$ by

$$
g(0)=2 \operatorname{Re}\left\langle A x_{0}, x_{0}\right\rangle, \quad g(t)=\frac{\ln \left(\left\|T(t) x_{0}\right\|^{2}\right)}{t}, \quad t>0 .
$$

$g$ has the following properties:

1. $g$ is continuous on $[0, \infty)$ and differentiable on $(0, \infty)$.

2. $g$ is nondecreasing. 
Proof. Part 1 is easy to see. Thus it remains to prove part 2 of the lemma. Since $x_{0}$ has norm one, we know that

$$
\int_{\mathbb{C}} 1 d\left\|E(\lambda) x_{0}\right\|^{2}=\left\|x_{0}\right\|^{2}=1 .
$$

We define $\Omega=\left\{\lambda \in \mathbb{C} \mid \operatorname{Re} \lambda \leq \lambda_{0}\right\}$ and $\mu(\omega)=\left\|E(\omega) x_{0}\right\|^{2}$, where $\omega$ is a Borel subset of $\mathbb{C}$. Thus $\mu(\Omega)=1$. If we denote $\left\|T(t) x_{0}\right\|^{2}$ by $\nu(t)$, then it is easy to see that

$$
\dot{g}(t)=\frac{t \cdot \dot{\nu}(t)-\nu(t) \ln (\nu(t))}{t^{2} \nu(t)}, \quad t>0 .
$$

Note that $\nu(t)$ is differentiable because $x_{0} \in D(A) . \nu(t)>0$ implies that the sign of $\dot{g}(t)$ is determined by the numerator. Using (14), the numerator is given by

$$
t \cdot \int_{\Omega} 2 \operatorname{Re}(\lambda) e^{2 \operatorname{Re}(\lambda) t} d \mu(\lambda)-\int_{\Omega} e^{2 \operatorname{Re}(\lambda) t} d \mu(\lambda) \cdot \ln \left(\int_{\Omega} e^{2 \operatorname{Re}(\lambda) t} d \mu(\lambda)\right) .
$$

Consider next the function

$$
\phi(x)=x \ln (x), \quad x>0 .
$$

It is easy to see that

$$
\ddot{\phi}(x)=\frac{1}{x}, \quad x>0 .
$$

The positivity of $\ddot{\phi}$ implies that $\phi$ is convex on $(0, \infty)$. Using Jensen's inequality (Lemma 3.1) with this choice of $\phi$ and $\mu$, and $f$ given by $f(\lambda)=e^{2 \operatorname{Re}(\lambda) t}$, we get

$$
\begin{array}{rl}
\int_{\Omega} e^{2 \operatorname{Re}(\lambda) t} & d \mu(\lambda) \cdot \ln \left(\int_{\Omega} e^{2 \operatorname{Re}(\lambda) t} d \mu(\lambda)\right) \\
& =\phi\left(\int_{\Omega} e^{2 \operatorname{Re}(\lambda) t} d \mu(\lambda)\right) \\
& \leq \int_{\Omega} \phi(f(\lambda)) d \mu(\lambda) \\
& =\int_{\Omega} e^{2 \operatorname{Re}(\lambda) t} \cdot 2 \operatorname{Re}(\lambda) t d \mu(\lambda) .
\end{array}
$$

Comparing this with (17), we conclude that $\dot{g}(t)$ is nonnegative for $t>0$, which shows that $g$ is nondecreasing.

For $x \in D(A) \backslash\{0\}$ and $s \geq 0$, we define the function $g_{x, s}:[0, \infty) \rightarrow \mathbb{R}$ by

$$
g_{x, s}(t):=\frac{1}{t} \ln \left(\frac{\|T(t) T(s) x\|^{2}}{\|T(s) x\|^{2}}\right),
$$

if $t>0$, and by

$$
g_{x, s}(0):=2 \frac{\operatorname{Re}\langle A T(s) x, T(s) x\rangle}{\|T(s) x\|^{2}} .
$$

We have the following useful corollary.

Corollary 3.3. We have that

1. the functions $g_{x, s}$ are continuous and nondecreasing on $[0, \infty)$ and differentiable on $(0, \infty)$; 
2. for $s_{2}>s_{1} \geq 0$ we have

$$
g_{x, s_{2}}(0) \geq g_{x, s_{1}}\left(s_{2}-s_{1}\right) .
$$

Proof. The first part of the corollary follows immediately from the previous lemma by taking $x_{0}:=\|T(s) x\|^{-1} T(s) x$. For $t>0$ we have

$$
\begin{aligned}
& g_{x, s_{2}}(t) \\
& =\frac{1}{t}\left[g_{x, s_{1}}\left(t+s_{2}-s_{1}\right)\left(t+s_{2}-s_{1}\right)-g_{x, s_{1}}\left(s_{2}-s_{1}\right)\left(s_{2}-s_{1}\right)\right] \\
& =g_{x, s_{1}}\left(t+s_{2}-s_{1}\right)+\frac{\left(s_{2}-s_{1}\right)}{t}\left[g_{x, s_{1}}\left(s_{2}-s_{1}+t\right)-g_{x, s_{1}}\left(s_{2}-s_{1}\right)\right] \\
& \geq g_{x, s_{1}}\left(t+s_{2}-s_{1}\right),
\end{aligned}
$$

where we have used part 1 . The result follows by continuity as $t$ tends to zero.

Lemma 3.4. Let $N \in \mathbb{N}$ and let $h$ be a nondecreasing function on the interval $\left[s_{0}, s_{N}\right]$. Assume that the values of $h$ lie in the interval $\left[\beta_{0}, \beta_{N}\right]$. If we have that $\left[s_{0}, s_{N}\right]$ is the union of the intervals $\left[s_{n}, s_{n+1}\right], n=0, \ldots, N-1$, and $\left[\beta_{0}, \beta_{N}\right]$ is the union of the intervals $\left[\beta_{n}, \beta_{n+1}\right], n=0, \ldots, N-1$, then there exists $n_{0} \in\{0, \ldots, N-1\}$ such that $h\left(\left[s_{n_{0}}, s_{n_{0}+1}\right]\right) \subset\left[\beta_{n_{0}}, \beta_{n_{0}+1}\right]$.

Proof. We prove this by induction on $N$. For $N=1$ it trivially holds. So assume that it holds for $N-1$.

If $h\left(\left[s_{0}, s_{1}\right]\right) \subset\left[\beta_{0}, \beta_{1}\right]$, then we are done. If this inclusion does not hold, then by the fact that $h$ is nondecreasing, $h\left(s_{1}\right)>\beta_{1}$. Hence $h\left(\left[s_{1}, s_{N}\right]\right) \subset\left[\beta_{1}, \beta_{N}\right]$. Now the intervals $\left[s_{1}, s_{N}\right]$ and $\left[\beta_{1}, \beta_{N}\right]$ are divided into $N-1$ subintervals, and by the induction assumption we conclude that the result holds.

We are now in a position to prove Theorem 1.3.

Proof of Theorem 1.3. Using the fact that the semigroup is normal and exponentially stable, there exists a constant $\alpha_{0}<0$ such that

$$
\left\|T(t) x_{0}\right\|^{2}=\int_{\mathbb{C}} e^{2 \operatorname{Re}(\lambda) t} d\left\|E(\lambda) x_{0}\right\|^{2} \leq e^{2 \alpha_{0} t}\left\|x_{0}\right\|^{2}
$$

for every $t \geq 0$ and every $x_{0} \in H$. We define the sequence $\left(\alpha_{n}\right)_{n \in \mathbb{N}_{0}}$ by

$$
\alpha_{n}=\alpha_{0}\left(1+\frac{\sqrt{m}}{5 e}\right)^{n}, \quad n \in \mathbb{N},
$$

where $m$ is the constant in (HT). An easy calculation shows that

$$
\frac{\alpha_{n+1}-\alpha_{n}}{\alpha_{n}}=\frac{\sqrt{m}}{5 e}<\frac{\sqrt{m}}{4 e} .
$$

We further define

$$
t_{n}:=\frac{1}{\alpha_{n}-\alpha_{n+1}}, \quad n \in \mathbb{N}_{0} .
$$

Thus Proposition 2.2 and Remark 2.3, with $\varepsilon \in\left(0,9 e^{2}\right)$ fixed, imply the following.

There is a constant $\kappa>0$ such that for every $x_{0} \in D(A)$ satisfying

$$
e^{\alpha_{n+1} t}\left\|x_{0}\right\| \leq\left\|T(t) x_{0}\right\| \leq e^{\alpha_{n} t}\left\|x_{0}\right\|, \quad 0 \leq t \leq t_{n},
$$


we have

$$
\kappa \int_{0}^{t_{n}}\left\|C T(t) x_{0}\right\|^{2} d t \geq\left\|x_{0}\right\|^{2}
$$

Note that $\kappa$ is independent of $n$.

Let $x_{0} \in D(A) \backslash\{0\}$. We define the function $g_{0}:[0, \infty) \rightarrow \mathbb{R}$ by

$$
g_{0}(t):=g_{x_{0}, 0}(t) .
$$

The function $g_{0}$ is nondecreasing, by Lemma 3.2, and there exists $N \in \mathbb{N}$, which may depend on $x_{0}$, such that

$$
2 \alpha_{N} \leq g_{0}(0)<2 \alpha_{N-1}
$$

Let

$$
s_{0}:=0, \quad s_{n}:=\sum_{j=1}^{n} t_{N-j}, \quad n \in\{1, \ldots, N\} .
$$

It is easy to see that the sequence $\left(s_{n}\right)_{n}$ is increasing and may depend on $x_{0}$. However, we have

$$
s_{n} \leq \sum_{j=0}^{\infty} t_{j}=: s_{\infty}<\infty,
$$

and $s_{\infty}$ does not depend on $n$ or $x_{0}$. Using the functions defined in (18) and (19), we define on the interval $\left[s_{0}, s_{N}\right]$ the function

$$
h(t)=g_{x_{0}, s_{n}}\left(t-s_{n}\right), \quad t \in\left[s_{n}, s_{n+1}\right), \quad n \in\{0, \ldots, N-1\} .
$$

By Corollary 3.3, the function $h$ is nondecreasing. Using (20) and (22), we have that $h\left(\left[s_{0}, s_{N}\right]\right) \subset\left[2 \alpha_{N}, 2 \alpha_{0}\right]$.

Defining $\beta_{n}=2 \alpha_{N-n}$, we obtain by Lemma 3.4 that for some $n_{0} \in\{0, \ldots, N-1\}$

$$
h\left(\left[s_{n_{0}}, s_{n_{0}+1}\right]\right) \subset\left[2 \alpha_{N-n_{0}}, 2 \alpha_{N-n_{0}-1}\right] .
$$

Using the definition of $h$ from (23), this is equivalent to

$$
2 \alpha_{N-n_{0}} \leq g_{x_{0}, n_{0}}(t) \leq 2 \alpha_{N-n_{0}-1}, \quad t \in\left[0, t_{N-n_{0}-1}\right] .
$$

This implies

$$
e^{2 \alpha_{N-n_{0}} t}\left\|T\left(s_{n_{0}}\right) x_{0}\right\|^{2} \leq\left\|T(t) T\left(s_{n_{0}}\right) x_{0}\right\|^{2} \leq e^{2 \alpha_{N-n_{0}-1} t}\left\|T\left(s_{n_{0}}\right) x_{0}\right\|^{2}
$$

for $t \in\left[0, t_{N-n_{0}-1}\right]$. Thus using (21) we obtain

$$
\kappa \int_{0}^{t_{N-n_{0}-1}}\left\|C T(t) T\left(s_{n_{0}}\right) x_{0}\right\|^{2} d t \geq\left\|T\left(s_{n_{0}}\right) x_{0}\right\|^{2} .
$$

Due to the fact that $s_{\infty} \geq s_{n_{0}}$ and

$$
\left\|T\left(s_{\infty}\right) x_{0}\right\|^{2} \leq e^{2 \alpha_{0}\left(s_{\infty}-s_{n_{0}}\right)}\left\|T\left(s_{n_{0}}\right) x_{0}\right\|^{2} \leq\left\|T\left(s_{n_{0}}\right) x_{0}\right\|^{2},
$$

we have

$$
\kappa \int_{0}^{s_{\infty}}\left\|C T(t) x_{0}\right\|^{2} d t \geq\left\|T\left(s_{\infty}\right) x_{0}\right\|^{2}, \quad x_{0} \in D(A) .
$$

Proof of Corollary 1.4. In [14] it is shown that (HT) is necessary for exact observability. Conversely, for $C_{0}$-groups the notions of final state observability and exact observability are equivalent, and thus the corollary follows from Theorem 1.3. 
4. The Hautus test for strongly stable systems. In [3] it is proved that if (HT) holds with $m=1$ for all $s \in \mathbb{C}$ and the semigroup $(T(t))_{t>0}$ is exponentially stable, then $(A, C)$ is exactly observable. We show that the same result holds under weaker conditions.

Proposition 4.1. Let $(T(t))_{t>0}$ be a strongly stable semigroup, and let (HT) hold for $m \geq 1$ and $\left(s_{k}\right)_{k \in \mathbb{N}} \subset(-\infty, 0)$ with $s_{k} \rightarrow-\infty, k \rightarrow \infty$. Then $(A, C)$ is exactly observable.

Proof. Without loss of generality, we may assume that $m=1$. For $x_{0} \in D(A)$, (HT) is equivalent to

$$
s_{k}^{2}\left\|x_{0}\right\|^{2}-s_{k}\left\langle A x_{0}, x_{0}\right\rangle-s_{k}\left\langle x_{0}, A x_{0}\right\rangle+\left\|A x_{0}\right\|^{2}-s_{k}\left\|C x_{0}\right\|^{2} \geq s_{k}^{2}\left\|x_{0}\right\|^{2} .
$$

Taking the limit $k \rightarrow \infty$, we obtain

$$
\left\langle A x_{0}, x_{0}\right\rangle+\left\langle x_{0}, A x_{0}\right\rangle \geq-\left\|C x_{0}\right\|^{2} .
$$

Replacing $x_{0}$ by $T(t) x_{0}$, we see that (24) implies

$$
\frac{d}{d t}\left\|T(t) x_{0}\right\|^{2} \geq-\left\|C T(t) x_{0}\right\|^{2} .
$$

Integrating both sides from $t=0$ to $t=t_{1}$ gives

$$
\left\|T\left(t_{1}\right) x_{0}\right\|^{2}-\left\|x_{0}\right\|^{2} \geq-\int_{0}^{t_{1}}\left\|C T(t) x_{0}\right\|^{2} d t .
$$

Since the semigroup is strongly stable, we conclude that

$$
\int_{0}^{\infty}\left\|C T(t) x_{0}\right\|^{2} d t \geq\left\|x_{0}\right\|^{2} .
$$

This concludes the proof.

The second part of this section is devoted to the proof of Theorem 1.5. We start with the following simple lemma, where $\mathbb{D}$ denotes the set $\{\rho \in \mathbb{C}|| \rho \mid<1\}$.

Lemma 4.2. Let $T \in \mathcal{L}(H)$ be an operator satisfying

$$
\|(\rho I-T) x\| \geq c(1-|\rho|)\|x\|, \quad \rho \in \mathbb{D}, x \in H,
$$

for some constant $c>0$ independent of $\rho$ and $x$. Then there exists a constant $m>0$ such that

$$
\|(\rho I-T) x\| \geq m \frac{1-|\rho|}{|1-\rho|}\|(I-T) x\|, \quad \rho \in \mathbb{D}, x \in H .
$$

Proof. For $\rho \in \mathbb{D}$ and $x \in H$ we have

$$
\begin{aligned}
\|(I-T) x\| & \leq\|(\rho I-T) x\|+|1-\rho|\|x\| \\
& \leq\|(\rho I-T) x\|+\frac{1}{c} \frac{|1-\rho|}{1-|\rho|}\|(\rho I-T) x\| \\
& \leq\left(1+\frac{1}{c}\right) \frac{|1-\rho|}{1-|\rho|}\|(\rho I-T) x\| . \quad \square
\end{aligned}
$$

The following proposition shows a discrete time version of Theorem 1.5, which is of independent interest. 
Proposition 4.3. There exists a contraction $T \in \mathcal{L}(H)$ such that

$$
\|(\lambda I-T) x\| \geq \frac{1}{2}(1-|\lambda|)\|x\|, \quad \lambda \in \mathbb{D}, x \in H,
$$

and

$$
\lim _{n \rightarrow \infty}\left\|T^{n} x\right\|=0, \quad x \in H .
$$

An even stronger version of this proposition can be found in Faddeev [4, Theorem 3]. We include a simplified proof which treats our situation.

Proof. As $H$ we choose $\ell^{2}(\mathbb{N})$. We define $T \in \mathcal{L}\left(\ell^{2}(\mathbb{N})\right)$ by

$$
(T x)_{n+1}:=\mu_{n} x_{n}, \quad(T x)_{1}=0, \quad x \in \ell^{2}(\mathbb{N}), n \in \mathbb{N},
$$

where the sequence $\left(\mu_{n}\right)_{n}$ will be defined later on. The operator $T$ now satisfies, for $\lambda \in \mathbb{D}$,

$$
\|(T-\lambda I) x\|^{2}=|\lambda|^{2}\|x\|^{2}+\sum_{j=1}^{\infty}\left|\mu_{n}\right|^{2}\left|x_{n}\right|^{2}-2 \operatorname{Re}\left(\lambda \sum_{j=1}^{\infty} \mu_{j} x_{j} \overline{x_{j+1}}\right) .
$$

Using

$$
\left|2 \operatorname{Re} \lambda \mu_{j} x_{j} \overline{x_{j+1}}\right| \leq \beta_{j}|\lambda| \mu_{j}^{2}\left|x_{j}\right|^{2}+\beta_{j}^{-1}|\lambda|\left|x_{j+1}\right|^{2}
$$

for $j \in \mathbb{N}, \beta_{j}>0$, we get

$$
\begin{aligned}
& \|(T-\lambda I) x\|^{2} \\
\geq & \sum_{j=1}^{\infty}\left(|\lambda|^{2}+\mu_{j}^{2}\right)\left|x_{j}\right|^{2}-\sum_{j=1}^{\infty}\left(\beta_{j}|\lambda| \mu_{j}^{2}\left|x_{j}\right|^{2}+\beta_{j}^{-1}|\lambda|\left|x_{j+1}\right|^{2}\right) \\
= & \sum_{j=1}^{\infty}\left(|\lambda|^{2}+\mu_{j}^{2}\right)\left|x_{j}\right|^{2}-\sum_{j=1}^{\infty} \beta_{j}|\lambda| \mu_{j}^{2}\left|x_{j}\right|^{2}-\sum_{j=2}^{\infty} \beta_{j-1}^{-1}|\lambda|\left|x_{j}\right|^{2} \\
= & \sum_{j=1}^{\infty}\left(|\lambda|^{2}+\mu_{j}^{2}-\beta_{j}|\lambda| \mu_{j}^{2}-\beta_{j-1}^{-1}|\lambda|\right)\left|x_{j}\right|^{2},
\end{aligned}
$$

where $\beta_{0}^{-1}=0$. Choosing

$$
\beta_{j}:=\frac{j+1}{j}, \quad \beta_{0}^{-1}=0, \quad \text { and } \quad \mu_{j}:=\frac{j}{j+1},
$$

we obtain

$$
\|(T-\lambda I) x\|^{2} \geq \sum_{j=1}^{\infty}\left(|\lambda|^{2}+\frac{j^{2}}{(j+1)^{2}}-|\lambda|\left(\frac{j}{j+1}+\frac{j-1}{j}\right)\right)\left|x_{j}\right|^{2} .
$$

It remains to show that

$$
x^{2}+\frac{j^{2}}{(j+1)^{2}}-x \frac{2 j^{2}-1}{j^{2}+j} \geq \frac{1}{4}(1-x)^{2}
$$


for every $j \in \mathbb{N}$ and every $x \in[0,1]$, or equivalently,

$$
\frac{3}{4} x^{2}+\left(\frac{j^{2}}{(j+1)^{2}}-\frac{1}{4}\right)-x\left(\frac{2 j^{2}-1}{j^{2}+j}-\frac{1}{2}\right) \geq 0
$$

for every $j \in \mathbb{N}$ and every $x \in[0,1]$. For $j=1$, inequality (26) reads as $x^{2} \geq 0$, which is of course true. Now let $j \in \mathbb{N}$ with $n \geq 2$. We define $\gamma_{j}:=\frac{j^{2}}{(j+1)^{2}}-\frac{1}{4}$, $\beta_{j}:=-\left(\frac{2 j^{2}-1}{j^{2}+j}-\frac{1}{2}\right)$, and $\alpha_{j}:=\frac{3}{4}$. Since $\alpha_{j}>0$, it remains to show that the polynomial $\alpha_{j} x^{2}+\beta_{j} x+\gamma_{j}$ has no real root, and this is the case if $\beta_{j}^{2}-4 \alpha_{j} \gamma_{j}<0$. We have

$$
\beta_{j}^{2}-4 \alpha_{j} \gamma_{j}=\left(\frac{2 j^{2}-1}{j^{2}+j}-\frac{1}{2}\right)^{2}-\frac{3 j^{2}}{(j+1)^{2}}+\frac{3}{4}=\frac{1+j-2 j^{2}}{(j+1)^{2} j^{2}}<0 .
$$

This shows (25) for every $j \in \mathbb{N}$ and every $x \in[0,1]$, and thus $\|(\lambda I-T) x\| \geq$ $\frac{1}{2}(1-|\lambda|)\|x\|$ for $\lambda \in \mathbb{D}$ and $x \in H$. Since $\left|\mu_{j}\right|<1, j \in \mathbb{N}$, it is easy to see that $T$ is a contraction. Finally, $\prod_{j=1}^{\infty} \mu_{j}=0$ implies $\lim _{n \rightarrow \infty}\left\|T^{n} x\right\|=0$ for every $x \in H$.

Proof of Theorem 1.5. Let $T$ be the operator given by Proposition 4.3. Since $T$ is power stable, we get that $1 \notin \sigma_{p}(T)$. By Lemma 4.2 there exists a constant $m>0$ such that

$$
\|(\rho I-T) x\| \geq m \frac{1-|\rho|}{|1-\rho|}\|(I-T) x\|, \quad \rho \in \mathbb{D}, x \in H .
$$

Let $A: D(A) \subset H \rightarrow H$ be defined by

$$
\begin{aligned}
A x & :=(T+I)(T-I)^{-1} x, \quad x \in D(A), \\
D(A) & :=R(T-I) .
\end{aligned}
$$

In Sz.-Nagy and Foias [11, page 142] it is shown that $A$ generates a strongly stable contraction semigroup. It remains to show that (4) holds. For $x \in D(A)$ and $s \in \mathbb{C}$ with $\operatorname{Re} s<0$, we have $x=(T-I) y$ for some $y \in H, s=\frac{\rho+1}{\rho-1}$ for some $\rho \in \mathbb{D}$, and

$$
\begin{aligned}
\|(s I-A) x\| & =\left\|\frac{\rho+1}{\rho-1}(T-I) y-(T+I) y\right\|=\frac{2}{|1-\rho|}\|(\rho I-T) y\| \\
& \geq \frac{2}{|1-\rho|^{2}} m(1-|\rho|)\|x\| \geq m \frac{1-|\rho|^{2}}{|1-\rho|^{2}}\|x\|=m|\operatorname{Re} s|\|x\| .
\end{aligned}
$$

We have constructed an example of a strongly stable contraction semigroup such that $(A, 0)$ satisfies (HT), whereas this system is (clearly) not approximately controllable. One might wonder whether a similar example is possible with a bounded $A$. In the following paragraph, we explain that this is not possible.

If $A$ is bounded, then there exists a point in the left half plane, which is in the resolvent set of $A$. Since for any $s \in \mathbb{C}_{-},\|(s I-A) x\|^{2} \geq m|\operatorname{Re}(s)|^{2}\|x\|^{2}$, this implies that $\mathbb{C}_{-} \subset \rho(A)$, and

$$
\left\|(s I-A)^{-1}\right\| \leq \frac{\sqrt{m}}{|\operatorname{Re}(s)|}, \quad s \in \mathbb{C}_{-} .
$$

By van Casteren [1] this implies that $A$ is similar to a unitary group, and hence it cannot be strongly stable. 
Note that the boundedness of $A$ was only used to have a nonempty intersection of the left half plane and the resolvent set. Thus the above reasoning still remains valid under the weaker assumption that $\rho(A) \cap \mathbb{C}_{-} \neq \emptyset$. We can prove the following sufficient condition for approximate observability.

Proposition 4.4. Let $A$ be the generator of a strongly stable $C_{0}$-semigroup $(T(t))_{t \geq 0}$, satisfying $\rho(A) \cap i \mathbb{R} \neq \emptyset$. Suppose that $C$ is an infinite-time admissible observation operator for $(T(t))_{t \geq 0}$ and that $(H T)$ holds. Then $(A, C)$ is approximately observable.

Proof. We define

$$
V:=\left\{x_{0} \in H \mid C T(t) x_{0}=0 \text { in } L^{2}(0, \infty)\right\} .
$$

Assuming that the system is not approximately observable, we have $V \neq\{0\}$. Since $C$ is an infinite-time admissible observation operator for $(T(t))_{t \geq 0}$, we have that $V$ is a closed subspace of $H$. Furthermore, it is easy to see that $V$ is $(T(t))_{t \geq 0}$ invariant. Thus $\left(\left.T(t)\right|_{V}\right)_{t \geq 0}$ is a $C_{0}$-semigroup on $V$ with generator $A_{V}:=\left.A\right|_{V}$. The Hautus test (HT) implies

$$
\left\|\left(s I-A_{V}\right) x\right\| \geq m|\operatorname{Re} s|\|x\|, \quad \operatorname{Re} s<0, \quad x \in D\left(A_{V}\right)=D(A) \cap V .
$$

Due to the fact that $A$ generates a strongly stable $C_{0}$-semigroup and $\rho(A) \cap i \mathbb{R} \neq \emptyset$, there exists $\lambda \in \rho_{\infty}(A) \cap \mathbb{C}_{-}$, where $\rho_{\infty}(A)$ denotes the connected component of $\rho(A)$ that is unbounded to the right. Now [2, page 260] shows $\lambda \in \rho\left(A_{V}\right)$, and (27) implies $\mathbb{C}_{-} \in \rho\left(A_{V}\right)$. Applying [17, Theorem 3.1] or [1], we have that the $C_{0}$-semigroup $\left(\left.T(t)\right|_{V}\right)_{t \geq 0}$ can be extended to a bounded group, which is in contradiction to the strong stability of $\left(\left.T(t)\right|_{V}\right)_{t \geq 0}$. Thus $V=\{0\}$, and this completes the proof.

Acknowledgment. We would like to thank Yuri Tomilov for mentioning to us the reference [4].

\section{REFERENCES}

[1] J. A. van Casteren, Operators similar to unitary or self-adjoint ones, Pacific J. Math., 104 (1983), pp. 241-255.

[2] K.-J. Engel And R. NAgel, One-Parameter Semigroups for Linear Evolution Equations, Grad. Texts in Math. 194, Springer-Verlag, New York, 2000.

[3] P. Grabowski and F. M. Callier, Admissible observation operators. Semigroup criteria of admissibility, Integral Equations Operator Theory, 25 (1996), pp. 182-198.

[4] M. M. FADDEEv, Contraction operators that are similar to isometric operators, Vestnik Leningrad. Univ. Mat. Mekh. Astronom, vyp. 4 (1987), pp. 31-36, 110 (in Russian).

[5] B. JaCOB AND J. R. PARTington, Admissibility of control and observation operators for semigroups: A survey, in Proceedings of IWOTA 2002, Current Trends in Operator Theory and Its Applications, Oper. Theory Adv. Appl. 149, J. A. Ball, J. W. Helton, M. Klaus, and L. Rodman, eds., Birkhäuser, Basel, 2004, pp. 199-221.

[6] B. Jacob, AND R. Schnaubelt, Observability of polynomially stable systems, Systems Control Lett., 56 (2007), pp. 277-284.

[7] B. JACOB AND H. ZWART, Exact observability of diagonal systems with a finite-dimensional output operator, Systems Control Lett., 43 (2001), pp. 101-109.

[8] B. JaCOB And H. ZwART, A Hautus test for infinite-dimensional systems, in Unsolved Problems in Mathematical Systems and Control Theory, V. Blondel and A. Megretski, eds., Princeton University Press, Princeton, NJ, 2004, pp. 251-255.

[9] B. JACOB AND H. ZWART, Counterexamples concerning observation operators for $C_{0^{-}}$ semigroups, SIAM J. Control Optim., 43 (2004), pp. 137-153.

[10] L. Miller, Controllability cost of conservative systems: Resolvent condition and transmutation, J. Funct. Anal., 218 (2005), pp. 425-444.

[11] B. Sz.-Nagy and C. Folas, Harmonic Analysis of Operators on Hilbert Spaces, Translated from the French and revised, North-Holland, Amsterdam-London, 1970. 
[12] B. Opic And A. Kufner, Hardy-type Inequalities, Pitman Res. Notes Math. Ser. 219, Longman Scientific \& Technical, Harlow, UK, 1990.

[13] W. Rudin, Functional Analysis, 2nd ed., Internat. Ser. Pure Appl. Math., McGraw-Hill, New York, 1991

[14] D. L. Russell and G. Weiss, A general necessary condition for exact observability, SIAM J. Control Optim., 32 (1994), pp. 1-23.

[15] G. WeIss, Admissible observation operators for linear semigroups, Israel J. Math., 65 (1989), pp. $17-43$.

[16] Q. Zhou And M. Yамамото, Hautus condition on the exact controllability of conservative systems, Internat. J. Control, 63 (1997), pp. 371-379.

[17] H. ZwART, On the invertibility and bounded extension of $C_{0}$-semigroups, Semigroup Forum, 63 (2001), pp. 153-160. 